\title{
ASTROMETRIC TESTS OF GALACTIC EVOLUTION
}

\author{
GERARD GILMORE \\ Institute of Astronomy, Madingley Rd, Cambridge, UK \\ gil@mail.ast.cam.ac.uk
}

\section{Introduction}

There are many fundamental aspects of Galactic structure and evolution which can be studied best or exclusively with high quality three dimensional kinematics. Amongst these we note as examples determination of the orientation of the stellar velocity ellipsoid, and the detection of structure in velocity-position phase space. The first of these is the primary limitation at present to reliable and accurate measurement of the Galactic gravitational potential. The second is a critical test of current standard models of Galactic formation and evolution.

\section{Measuring Gravitational Potentials}

The classical method of measuring mass utilises the motions of stellar tracers in the Galaxy. This essentially measures the gravity-pressure-angular momentum balance of a suitable dynamically-relaxed tracer population. Appropriate analysis allows the gravitational force to be derived, and from this, via Poisson's equation, the mass density that generates that potential. All such analyses are applications of the collisionless Boltzmann equation. All are in practice approximate. The primary limitation in present analyses is the absence of accurate 3-dimensional kinematics, of the type which would be provided by a high precision astrometric project.

\subsection{THE COLLISIONLESS BOLTZMANN EQUATION}

The dynamics of any large stellar system are governed by the collisionless Bolzmann equation

$$
\frac{D f}{D t} \equiv \frac{\partial f}{\partial t}+\frac{\partial \vec{x}}{\partial t} \cdot \frac{\partial f}{\partial \vec{x}}+\frac{\partial \vec{v}}{\partial t} \cdot \frac{\partial f}{\partial \vec{v}}=0,
$$


where $f$ is the phase space density at the point $(\vec{x}, \vec{v})$ in phase space (ie there are $f(\vec{x}, \vec{v}) d^{3} \vec{x} d^{3} \vec{v}$ stars in a volume of size $d^{3} \vec{x}$ centered on $\vec{x}$ with velocity in the volume of size $d^{3} \vec{v}$ about $\vec{v}$ ).

The collisionless Boltzmann equation is satisfied by any stellar population, whether other stars are present or not. This arises because stars do not interact except through long-range gravity forces, and those are being described through a smooth background potential. Consequently, $f$ does not have to describe the entire Galaxy; one can concentrate on any subsample of stars, and apply the collisionless Boltzmann equation to it. Such subsamples are tracer populations, since one may use their kinematics to trace the potential of the Galaxy, irrespective of what generates this potential.

If one has a steady-state tracer population, and a time-independent potential, as the large-scale field in the Milky Way apparently is to an adequate approximation for the present purpose, then

$$
\frac{\partial f}{\partial t}=0 \text {. }
$$

While the Galaxy is not rotationally symmetric, the essential features of the present analysis do not depend on this asymmetry, so it may be suppressed for now. It is convenient to write out the collisionless Boltzmann equation in cylindrical polar coordinates $(r, \phi, z)$ in which $z=0$ is the disk plane of symmetry, with corresponding velocity components $\left(v_{r}, v_{\phi}, v_{z}\right)$ :

$$
v_{r} \frac{\partial f}{\partial r}+v_{z} \frac{\partial f}{\partial z}+\left(\mathcal{K}_{r}+\frac{v_{\phi}^{2}}{r}\right) \frac{\partial f}{\partial v_{r}}-\frac{v_{r} v_{\phi}}{r} \frac{\partial f}{\partial v_{\phi}}+\mathcal{K}_{z} \frac{\partial f}{\partial v_{z}}=0
$$

where the accelerations $\dot{v}_{r}, \dot{v}_{\phi}, \dot{v}_{z}$ explicit in the Boltzmann equation have been equated to the forces (real and fictitious) that cause them, and $\phi$ gradients in $f$ and in the potential set to zero. The vector $\overrightarrow{\mathcal{K}}(r, z)$ is the gravity force. Then clearly knowledge of $f(\vec{x}, \vec{v})$ allows the force components $\mathcal{K}_{r}$ and $\mathcal{K}_{z}$ to be derived. Note, though, that a general function $f$ will not allow a unique solution for $\mathcal{K}_{r}$ and $\mathcal{K}_{z}: f$ has five independent variables (we suppressed any $\phi$-dependence) and so cannot in general be made to satisfy the equation above, which contains only two functions of two variables. Since the equation cannot easily be solved in general with real data, one simplifies the analysis ani proceeds by taking velocity moments. Multiplying through by $v_{z}$ and by $v_{r}$ and integrating over all velocity space produces Jeans' equations:

$$
\begin{aligned}
\nu \mathcal{K}_{z} & =\frac{\partial}{\partial z}\left(\nu \sigma_{z z}^{2}\right)+\frac{1}{r} \frac{\partial}{\partial r}\left(R \nu \sigma_{r z}^{2}\right) \\
\nu \mathcal{K}_{r} & =\frac{1}{r} \frac{\partial}{\partial r}\left(r \nu \sigma_{r r}^{2}\right)+\frac{\partial}{\partial z}\left(\nu \sigma_{r z}^{2}\right)-\frac{\nu \sigma_{\phi \phi}^{2}}{r}
\end{aligned}
$$


where $\nu(r, z)$ is the space density of the stars, and $\overrightarrow{\vec{\sigma}}(r, z)$ their velocity dispersion tensor $\left(i e \sigma_{i j}^{2}=\left\langle v_{i} v_{j}\right\rangle\right)$. In this way we have separated the two force components (radial and vertical in this case), and can in principle derive them both from measurements of the moments of the velocity distributions and density of a stellar tracer population.

\subsection{THE TILT TERM}

The term involving $\sigma_{r z}$ in the Jeans equation is related to the tilt of the velocity ellipsoid in the $(\mathrm{r}, z)$ plane. It is our lack of knowledge of this term which is the greatest limitation in determination of mass distributions in the Galaxy. Two specific examples will suffice to illustrate this. This term has been treated explicitely in derivation of the surface mass density and scale length of the Galactic disk (Kuijken \& Gilmore 1989abc,1991; Fux \& Martinet 1994; Cuddeford \& Amendt 1992), which utilize eqn (4). It has also been discussed in terms of eqn (5), for the disk asymmetric drift by Gilmore, Wyse \& Kuijken (1989), and when relating the shape of the stellar distribution in the galactic halo to the shape of the distribution of dark matter by (for example) van der Marel (1991).

The two limiting cases may be discussed analytically, and obviously include orientation of the stellar velocity ellipsoid in cylindrical or in spherical polar coordinates. As an example, Kuijken \& Gilmore assumed the long axis of the velocity ellipsoid always points towards the centre of the Galaxy, as is appropriate for a round potential. In this case, they could treat the $\sigma_{r z}$ term as an extra force term. If the Galaxy's disk, like those of other spirals, has a vertical scale height which is constant with radius and a radially exponential surface density profile $\mu \propto e^{-r / h_{\mathrm{r}}}$, (see Fux \& Martinet 1994) then $\sigma_{z z} \propto \mu \propto e^{-r / \mathrm{h}_{\mathrm{r}}}$, and $\nu \propto \mu \propto e^{-r / \mathrm{h}_{\mathrm{r}}}$. from this Kuijken \& Gilmore were able to derive a relationship between $\sigma_{r z}$ and $\sigma_{z z}$. Since the details of this derivation have not previously been published, we present them here.

\subsection{DERIVATION OF THE TILT TERM $\sigma_{R Z}$}

Define the coordinate frames as:

Cylindrical polar $(r, z, \phi)$, with $\phi$ suppressed by symmetry, and with velocity components $\dot{z}$ and $\dot{r}$;

Spherical polar, $(\mathrm{R}, \theta, \phi)$, where we make $\theta$ the angle subtended at the Galactic centre between the radius vector $\mathrm{R}$ and the radial planar coordinate $r$, so that $\tan \theta=z / r$, and suppress $\phi$ by symmetry. The relevant velocity components, to avoid too many levels of subscripts, are $(A, B, C)$, with $B$ suppressed by symmetry. Thus, the $A$ component of velocity is the radial velocity away from the Galactic centre, the $C$ component is perpendicular to it, and at $z=0$ one has $\dot{r} \equiv A$, and $\dot{z} \equiv C$. 
The coordinate transformations by simple trigonometry are:

$$
\begin{aligned}
& \dot{z}=A \sin \theta+C \cos \theta \\
& \dot{r}=A \cos \theta-C \sin \theta
\end{aligned}
$$

By definition of the orientation of the velocity ellipsoid, $\operatorname{Cov}(A, C)=0$, and

$$
\begin{aligned}
\sigma_{r z} & =\operatorname{Cov}(\dot{r}, \dot{z}) \\
& =(\dot{z}-\langle\dot{z}\rangle)(\dot{r}-\langle\dot{r}\rangle) \\
& =\dot{z} \dot{r} \\
\dot{z} \dot{r} & =(A \sin \theta+C \cos \theta)(A \cos \theta-C \sin \theta) \\
& =A^{2} \sin \theta \cos \theta-C^{2} \cos \theta \sin \theta(+A C \text { terms }) \\
\Longrightarrow \sigma_{r z} & =\sin \theta \cos \theta\left(\sigma_{A A}-\sigma_{C C}\right) \\
\dot{z} \dot{z} & =(A \sin \theta+C \cos \theta)(A \sin \theta+C \cos \theta) \\
& =A^{2} \sin ^{2} \theta+C^{2} \cos ^{2} \theta(+A C \text { terms }) \\
\Longrightarrow \sigma_{z z} & =\sigma_{A A} \sin ^{2} \theta+\sigma_{C C} \cos ^{2} \theta
\end{aligned}
$$

Defining $\sigma_{A A}=\alpha^{2} \sigma_{C C}$ gives

$$
\sigma_{z z}=\left(\alpha^{2} \sin ^{2} \theta+\cos ^{2} \theta\right) \sigma_{C C}
$$

and

$$
\begin{aligned}
\sigma_{r z} & =\sin \theta \cos \theta\left(\alpha^{2}-1\right) \sigma_{C C} \\
& =\frac{\sin \theta \cos \theta\left(\alpha^{2}-1\right)}{\alpha^{2} \sin ^{2} \theta+\cos ^{2} \theta} \sigma_{z z}
\end{aligned}
$$

(Note: At $z=0$ of course $A \equiv \dot{r}$, and $C \equiv \dot{z}$, so $\sigma_{r r}=\alpha^{2} \sigma_{z z}$, but this cannot be true in general.)

Since $\tan \theta=z / r, \sin \theta \propto z, \cos \theta \propto r$, and hence

$$
\sigma_{r z}=\frac{r z\left(\alpha^{2}-1\right)}{\left(\alpha^{2} z^{2}+r^{2}\right)} \sigma_{z z}
$$

This then leads directly to:

$$
\begin{aligned}
\frac{1}{\nu r} \frac{\partial}{\partial r}\left(r \nu \sigma_{r z}\right) & =6 \sigma_{z z}\left\{\frac{4 z^{3}}{\left(4 z^{2}+r^{2}\right)^{2}}-\frac{r z}{\mathrm{~h}_{\mathrm{r}}\left(4 z^{2}+r^{2}\right)}\right\} \\
& =\mathrm{T}(r, z) \sigma_{z z}
\end{aligned}
$$

The vertical Jeans' equation now becomes

$$
\mathcal{K}_{z, \text { eff }}=\mathcal{K}_{z}-\mathrm{T}(r, z) \sigma_{z z}=\frac{1}{\nu} \frac{\partial}{\partial z}\left(\nu \sigma_{z z}\right)
$$


Given a 'true' $\mathcal{K}_{z}$, this linear equation can be solved for $\sigma_{z z}$ (especially since $\mathrm{T}$ has an analytic $z$-integral), and hence the effective force $\mathcal{K}_{z \text {,eff }}$ calculated, with its corresponding potential. Kuijken \& Gilmore were then able to proceed by assuming, as a first approximation, that the tracer population moved under the action of this potential.

Given the quality of observational data currently available relevant to the determination of the Galactic $\mathcal{K}_{z}$ force law, the validity or otherwise of this assumption is the primary uncertainty in present determinations of the mass distribution in the optical parts of the Galaxy. The true orientation of the stellar ellisoid could be measured directly from suitable precise astrometric data. Data for a large number of stars at distances of up to $2 \mathrm{kpc}$, with a space motion precision of about $1 \mathrm{~km} / \mathrm{s}$ are required. Sub-milliarcsec astrometry can realize this precision. We would then have a precise, reliable, and assumption-free direct determination of that part of the total Galactic mass density associated with the Galactic disk, and that part distributed in a (dark) halo.

\section{The Shape of the Dark Halo}

Arguments similar to those above, but applied to the radial Jeans equation (5) have been outlined by van der Marel (1991). He applied the methodology to determination of the shape of the dark matter distribution. The observational constraints are radial velocity data at several locations and star count determinations of the shape of the stellar distribution. The most recent and extensive determination of the shape of the stellar halo, which is in good agreement with most earlier determinations, provides $c / a=0.55$ (Larsen \& Humphries 1994).

Van der Marel shows that this observed shape of the stellar halo implies a corresponding axis ratio $c / a \approx 0.25$ for the case of an adopted spherical alignment of the velocity ellipsoid, and an axis ratio $c / a \approx 0.55$ for the case of an adopted cylindrical alignment of the stellar velocity ellipsoid. That is, there is more than a factor of two uncertainty generated by the absence of 3-D kinematics. This fractional error has the same effect as an error of a factor of two in the observed shape of the stellar halo, an error bound substantially outside current observational limits. That is, our best determinations of the shape of the dark matter halo in the Galaxy from a mix of stellar distribution and (radial velocity) kinematic data are entirely dominated by missing suitably precise astrometric data. These limits could be removed by provision of data with a precision of a few $\mathrm{km} / \mathrm{s}$ for stars at distances of $5-10 \mathrm{kpc}$. 


\section{Tests of Galactic Formation and Evolution}

Modern models of Galaxy formation make fairly specific predictions which are amenable to detailed test with Galactic kinematic and chemical abundance data. For example, popular Cold Dark Matter models 'predict' growth of the Galaxy about a central core, which should contain the oldest stars. Later accretion of material forms the outer halo and the disks, while continuing accretion will continue to affect the kinematic structure of both the outer halo and the thin disk.

This blend-and-stir process will have been common at high redshifts, when a rain of dwarf 'proto-galaxies' was normal weather for a budding giant. It continues today, at a rate which may still be significant for some galaxies. The term 'significant' here is worth some thought: in the central regions of galaxies masses are large, timescales are short and dynamical friction effective. Thus significant changes to a galaxy require mergers of components of comparable mass. It has been suggested that such mergers would destroy the thin disk of a galaxy like the Milky Way, as argued recently by Toth \& Ostriker(1992). If this argument were correct then normal late-type spirals must have completed the bulk of their merger events at very early times.

In the outer parts of galaxies mass densities are low, the fraction of the total luminous galaxy which is seen is very small, and timescales are comparable to a Hubble time. Thus one expects relatively little fossil kinematic structure to be observble in the central regions of normal galaxies, but it is probable that a large fraction of the outer parts of a large galaxy is a recent (on kinematic timescales) acquisition from afar. Fundamentally, the central regions of a galaxy need not be related in any obvious way to the outer parts of that same galaxy.

This picture, which contains aspects of both the monolithic ('ELS') and the multi-fragment ('Searle-Zinn') pictures often discussed in chemical evolution models, makes some specific predictions which are amenable to test. One specific example of current interest is the 'prediction' that mergers of small satellites are an essential feature of galactic evolution. This leads one to look for kinematic and spatial structures, and 'moving groups', as a primary test of such models. That is, the galaxy formation concepts outlined above suggest that a considerable amount of structure in the phase space distribution function for those stars (and DM particles) which inhabit the outer reaches of the galaxy is to be expected. This structure will be the remnant dispersion orbits occupied by the debris of former galactic satellites and near-neighbours which have now lost their former isolated identity. The existence of this structure provides a challenge in two ways: to devise dynamical analysis methods and/or sample selection methods which will 
still provide a 'fair sample' of the outer galaxy for dynamical studies; and to identify the fractional amount of phase space substructure, if any, and so test the (CDM) galaxy merger models.

The existence of such phase space structure in stars of the thick disk and halo, in addition to the younger stellar populations, has been persuasively argued by Eggen for many years (cf Eggen 1987 for a review). Such moving groups are of course a specific high contrast example of the structure being considered here.

Direct evidence for an ongoing merger event has been discovered recently in a study by Ibata, Gilmore \& Irwin (1994). While investigating the kinematic structure of the Galactic bulge, they discovered a large phase-space structure consisting of $>100 \mathrm{~K}$ giant, $\mathrm{M}$ giant and carbon stars in three low Galactic latitude fields. The group has a velocity dispersion of $<10 \mathrm{kms}^{-1}$, and a mean heliocentric radial velocity of 140 $\mathrm{kms}^{-1}$, that varies by less than $5 \mathrm{kms}^{-1}$ over the $8^{\circ}$ wide region of sky that the three kinematic fields cover. In a subsequent determination of the colour-magnitude relationship for that line of sight, a giant branch, red horizontal clump and horizontal branch are clearly visible, superimposed on the general distribution made up of stars in the bulge, and in the Galactic foreground. Stars belonging to the low velocity dispersion group lie on the upper giant branch of the colour-magnitude relation. From the magnitude of the horizontal branch, Ibata, Gilmore \& Irwin find that the object is situated $15 \pm 2 \mathrm{kpc}$ from the Galactic centre.

An isodensity map shows an object which is elongated (with axial ratio $\approx 3$ ), spanning $>10^{\circ}$ on the sky in a direction perpendicular to the Galactic plane. The interpretation is clear: this is a discovery, in kinematic phase space, of a dwarf (former) satellite galaxy currently well inside the galactic optical boundary. The tidal radius of the dwarf galaxy is then approximately an order of magnitude smaller than its apparent size on the sky, so most of its members will disperse into the Galactic halo over the next $\approx 10^{8}$ years. This finding clearly supports galaxy formation scenarios of with significant merging events happening right up to the present epoch.

The implications for the present are substantial. Kinematics can and has discovered phase space structure; presumably much more remains to be found. Kinematics can determine the present and future orbits of the (former) member stars of this dwarf galaxy. Kinematics can map out the merger history of the Milky Way. When astrometric data can provide distances to a few percent, and kinematics to a few $\mathrm{km} / \mathrm{s}$, at distances up to $20 \mathrm{kpc}$ from the Sun, then we will be able to determine in detail the evolutionary history, and the three-dimensional distribution of mass, in the Galaxy. 


\section{References}

Cuddeford, P. \& Amendt P. (1992) MNRAS 256166

Eggen, O.J. (1987) in The Galaxy, eds G. Gilmore \& R. Carswell, p211 (Reidel)

Fux, R. \& Martinet, L. (1994), A\&A, 287 L21

Ibata, R., Gilmore, G., \& Irwin, M. (1994) Nature 370194

Gilmore, G., Wyse, R.F.G., \& Kuijken, K. (1989) ARAA 27555

Kuijken, K. \& Gilmore, G. (1989a) MNRAS 239571

Kuijken, K. \& Gilmore, G. (1989b) MNRAS 239605

Kuijken, K. \& Gilmore, G. (1989c) MNRAS 239651

Kuijken, K. \& Gilmore, G. (1991) Ap.J. 367 L9

Larsen, J.A. \& Humphries, R. M. (1994) preprint

van der Marel, R.P. (1991), MNRAS, 248515

Toth, G., \& Ostriker, J. (1992) Ap.J. 3895. 